\title{
Análise de parâmetros $\pi$-híbridos de um MOSFET com encapsulamento TO-220 sob feixes de raios X para diagnóstico médico
}

Analysis of hybrid- $\pi$ parameters of a MOSFET with TO-220 encapsulation under X-ray beams for medical diagnostic

\author{
D. S. Monte ${ }^{1 *}$ L. A. P. Santos ${ }^{2}$ \\ ${ }^{1}$ Departamento de Energia Nuclear, Universidade Federal de Pernambuco, Recife-PE, Brasil
}

${ }^{2}$ Laboratório de Instrumentação Nuclear, Centro Regional de Ciências Energéticas e Nucleares, Comissão Nacional de Energia Nuclear, 50740-540, Recife-PE, Brasil

*soaresmonte@gmail.com

(Recebido em 19 de novembro de 2020; aceito em 17 de dezembro de 2020)

\begin{abstract}
Nas últimas décadas o MOSFET (Metal Oxide Semiconductor Field Effect Transistor) tem sido utilizado como detector de radiação ionizante, principalmente em aplicações dosimétricas em radioterapia. A base do método de funcionamento de tal dispositivo eletrônico em radioterapia consiste em mensurar a variação da tensão de limiar do MOSFET, pois este parâmetro elétrico é proporcional à dose de radiação ionizante depositada no dispositivo. O objetivo deste trabalho é apresentar um método inovador de medição da dose de radiação ionizante por meio da medição da variação de alguns parâmetros $\pi$-híbridos de um MOSFET. Foram analisados apenas transistores com encapsulamento TO-220 e dois tipos de polaridades distintas: canal $n$ e canal $p$. Os dispositivos foram submetidos aos feixes de raios X cuja energia está na faixa aplicada ao diagnóstico médico. Os resultados mostraram que os parâmetros $\pi$-híbridos transcondutância e uma capacitância variam com a dose acumulada de radiação até o limite de 100 Gy.

Palavras-chave: MOSFET, parâmetro $\pi$-híbrido, raios X
\end{abstract}

In recent decades, MOSFET (Metal Oxide Semiconductor Field Effect Transistor) has been used as an ionizing radiation detector, mainly in dosimetric applications in radiotherapy. The basis of the method of operation of such an electronic device in radiotherapy consists of measuring the variation of the threshold voltage of the MOSFET, since this electrical parameter is proportional to the dose of ionizing radiation deposited in the device. The objective of this work is to present an innovative method of measuring the dose of ionizing radiation by measuring the variation of some hybrid $-\pi$ parameters of a MOSFET. Only transistors with TO-220 encapsulation and two different polarity types were analyzed: $n$ channel and $p$ channel. The devices were submitted to X-ray beams whose energy is in the range applied to medical diagnosis. The results showed that the hybrid- $\pi$ parameters transconductance and a capacitance varied with the accumulated dose up to the limit of $100 \mathrm{~Gy}$.

Keywords: MOSFET, hybrid- $\pi$ parameter, $\mathrm{X}$ ray

\section{INTRODUÇÃO}

O MOSFET (Metal Oxide Semiconductor Field Effect Transistor) é um dispositivo eletrônico que possui variadas aplicações, sendo um dos principais responsáveis pelo desenvolvimento tecnológico vivenciado na atualidade proporcionado pelo avanço da eletrônica. O MOSFET possui basicamente três terminais, a saber: porta $(\mathrm{G})$, fonte (S), dreno (D). Esse dispositivo é normalmente utilizado em circuitos eletrônicos os mais diversos [1, 2]. Além disso, desde a década de 1980 o MOSFET é utilizado como detector de radiação ionizante, como dosímetro em radioterapia. $\mathrm{O}$ método para medir a dose em radioterapia é baseado na correlação entre a variação da tensão de limiar do MOSFET com a dose acumulada de radiação recebida pelo dispositivo [3-8]. A tensão de limiar é um parâmetro elétrico característico do MOSFET.

Neste trabalho pretende-se desenvolver um método inovador para mensuração da dose de radiação X com base em parâmetros elétricos diferentes da tensão de limiar que é o convencional quando se refere ao MOSFET. O método proposto aqui está fundamentado na análise de parâmetros 
elétricos que caracterizam o MOSFET quando está sendo empregado na amplificação de sinais elétricos de baixa intensidade (pequenos sinais). Nesta condição de uso, o MOSFET tem seu comportamento modelado por circuitos elétricos equivalentes compostos por elementos como resistores, capacitores e fontes de corrente controladas por tensão [1]. Um dos principais modelos utilizados pelos projetistas de circuitos é o modelo $\pi$-híbrido, cujos elementos constituintes são conhecidos como parâmetros $\pi$-híbridos [9]. A Figura 1 apresenta o modelo adotado neste trabalho para análise dos parâmetros $\pi$-híbridos do MOSFET. Quando o MOSFET é submetido à radiação ionizante, tais parâmetros podem variar e então ser correlacionados com a dose recebida pelo dispositivo.

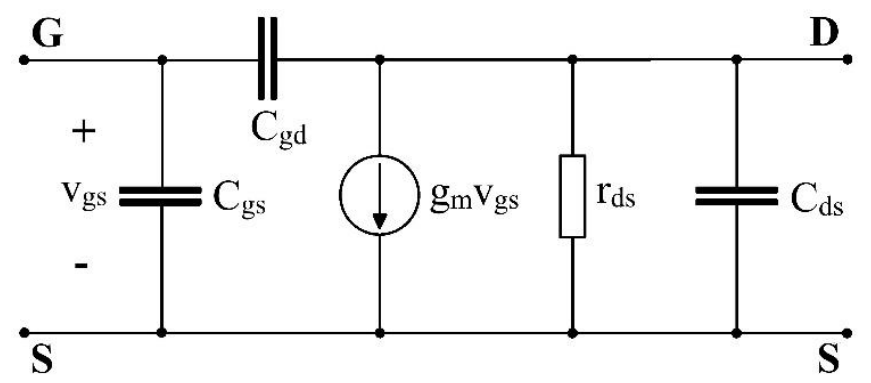

Figura 1: Modelo $\pi$-híbrido para o MOSFET.

\section{MATERIAL E MÉTODOS}

Neste trabalho, os parâmetros $\pi$-híbridos selecionados para serem avaliados foram: a transcondutância $\left(g_{m}\right)$, a capacitância entre a porta e o dreno $\left(C_{g d}\right)$ e a capacitância entre o dreno e a fonte $\left(C_{d s}\right)$. Estas capacitâncias não têm comportamento linear e é função da estrutura, da geometria e das tensões de polarização do MOSFET [10].

A medição dos parâmetros $\pi$-híbridos foi realizada utilizando-se o medidor LCR Agilent ${ }^{\circledR}$ 4263B. Um medidor LCR consiste em um instrumento que mensura os seguintes parâmetros elétricos: indutância $(\mathrm{L})$, capacitância $(\mathrm{C})$ e resistência $(\mathrm{R})$. Ao medidor LCR foram acoplados dois acessórios: o adaptador de tensão de polarização externa $16065 \mathrm{C}$ e o acessório de teste 16047E, ambos de marca Agilent ${ }^{\circledR}$. Uma unidade de alimentação e medição de precisão Agilent ${ }^{\circledR}$ B2901A foi conectada ao adaptador $16065 \mathrm{C}$ para fornecimento das tensões de polarização externas. Os MOSFETs selecionados para os experimentos estão listados na Tabela 1. A conexão dos transistores ao medidor LCR para medição dos parâmetros $\pi$-híbridos é apresentada na Figura 2 [11-14].

Tabela 1: MOSFETs avaliados nos experimentos.

\begin{tabular}{cccc}
\hline \multirow{2}{*}{ Série } & \multicolumn{3}{c}{ MOSFETs } \\
\cline { 2 - 4 } FQP20N06 & Canal $n$ & Encapsulamento & Características Elétricas \\
& & & $\mathrm{I}_{\text {Dmáx. }}=20 \mathrm{~A}$ \\
FQP17P10 & Canal $p$ & TO-220 & $\mathrm{V}_{\text {DSmáx. }}=60 \mathrm{~V}$ \\
& & $\mathrm{I}_{\text {Dmáx. }}=-16,5 \mathrm{~A}$ \\
& & & $\mathrm{~V}_{\text {DSmáx. }}=-100 \mathrm{~V}$ \\
\hline
\end{tabular}

Os transistores foram irradiados com feixes de raios $\mathrm{X}$ em doses quantizadas de 20 Gy no intervalo de 0 a 100 Gy como proposto por Gonçalves Filho et al. (2018) [15]. O equipamento gerador de raios $\mathrm{X}$ utilizado foi o Pantak ${ }^{\circledR} \mathrm{HF} 160$, cuja configuração de funcionamento foi ajustada para $100 \mathrm{kV}$ e $10 \mathrm{~mA}$. Os transistores foram posicionados a uma distância $d=10 \mathrm{~cm}$ em relação ao foco do equipamento gerador de raios X. O equipamento Pantak ${ }^{\circledR} \mathrm{HF}-160$ tem filtração inerente de $0,8 \mathrm{~mm}$ de berílio e por isso uma filtração adicional de $1,0 \mathrm{~mm}$ de alumínio foi utilizada como proposto nos trabalhos de Gonçalves Filho et al. (2018) [15] e Santos et al. (2013) [16]. A Figura 
3 apresenta o arranjo experimental empregado neste trabalho. Para realização dos procedimentos de irradiação dos MOSFETs a taxa de dose de $222 \mathrm{mGy} / \mathrm{s}$ foi utilizada em todos os experimentos realizados.

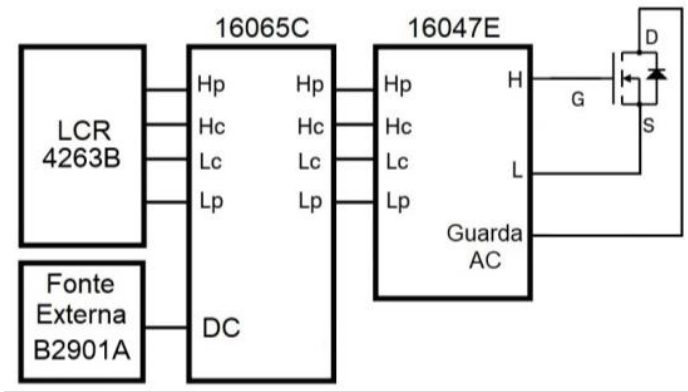

(a)

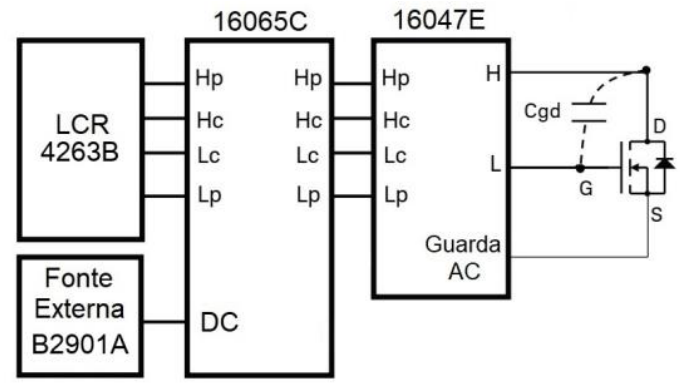

(b)

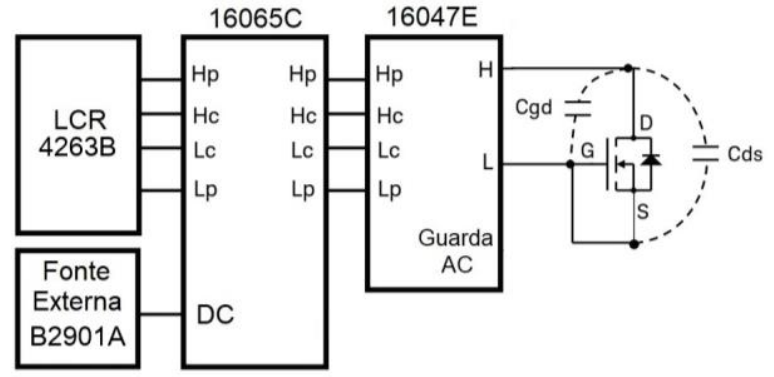

(c)

Figura 2: Configurações utilizadas para medição dos parâmetros $\pi$-híbridos: (a) $g_{m},(b) C_{g d} e(c) C_{d s}$.

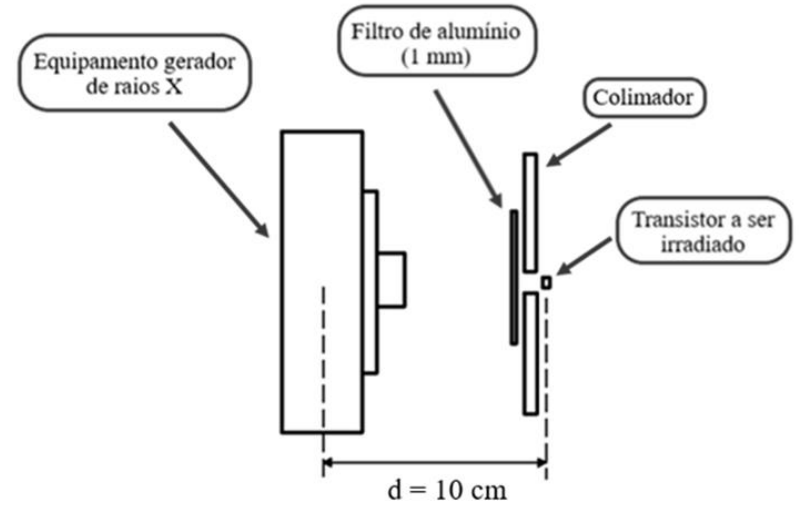

Figura 3: Arranjo experimental utilizado para irradiação dos transistores.

As medições dos parâmetros $\pi$-híbridos selecionados foram realizadas após intervalo de 60 minutos contados a partir do término de cada procedimento de irradiação. A escolha deste intervalo de tempo decorre do fato de que são necessários pelo menos 30 minutos após a irradiação para que ocorra um rearranjo (estabilização) dos defeitos induzidos pela irradiação no cristal de silício [17, 18], fenômeno este conhecido como annealing [19-21]. A temperatura ambiente do laboratório era controlada $\left(25 \pm 1^{\circ} \mathrm{C}\right)$ e tal efeito não foi uma variável de perturbação. Os transistores foram irradiados em duas condições distintas: na ausência de polarização e sob polarização elétrica. A polarização adotada foi $V_{G S}=0 \mathrm{~V}$ e $V_{D S}=10 \mathrm{~V}$ para os MOSFETs canal $n$ e $V_{G S}=0 \mathrm{~V}$ e $V_{D S}=-10$ $\mathrm{V}$ para os MOSFETs canal $p$. Os resultados obtidos nessas duas condições foram comparados e analisados. Para cada modelo de transistor listado na Tabela 1 foram irradiadas amostras em triplicata, somando um total de 12 transistores. São utilizados para os gráficos a média de 3 leituras 
de cada triplicata, perfazendo um total de 9 pontos amostrais para cada triplicata. Desse total de 12 transistores têm-se: seis MOSFETs canal $n$ e seis MOSFETs canal $p$. Em cada conjunto de seis transistores foram irradiados três na ausência de polarização e três sob polarização elétrica.

A medição de cada um dos parâmetros foi realizada estando o medidor LCR configurado para um sinal de teste $V_{c a}=20 \mathrm{mV}_{\text {rms }}$ e frequência de teste $f=100 \mathrm{kHz}$. As condições de medição da transcondutância $g_{m}$ foram: $V_{D S}=0 \mathrm{~V}$ e $V_{G S}$ variando no intervalo de 0 a $5 \mathrm{~V}$ em incrementos de $0,1 \mathrm{~V}$ para o MOSFET canal $n$ e $V_{D S}=0 \mathrm{~V}$ e $V_{G S}$ variando no intervalo de -1 a $-6 \mathrm{~V}$ em incrementos de $-0,1$ V para o MOSFET canal $p$. No caso das capacitâncias $C_{g d}$ e $C_{d s}$, as condições de medição foram: $V_{G S}=0 \mathrm{~V}$ e $V_{D S}$ variando no intervalo de 0 a $25 \mathrm{~V}$ em incrementos de $1,0 \mathrm{~V}$ para o MOSFET canal $n$ e $V_{G S}=0 \mathrm{~V}$ e $V_{D S}$ variando no intervalo de 0 a $-25 \mathrm{~V}$ em incrementos de $-1,0 \mathrm{~V}$ para o MOSFET canal $p$.

\section{RESULTADOS E DISCUSSÃO}

Os resultados obtidos para as medições da transcondutância $g_{m}$ originaram as curvas $g_{m} \times V_{G S}$ apresentadas na Figura 4. Em todas as condições analisadas ocorre o deslocamento das curvas no sentido negativo do eixo das abscissas à medida que a dose acumulada aumenta. Comparando-se os resultados para os transistores do mesmo tipo que foram irradiados na ausência de polarização e sob polarização elétrica, nota-se que há diferença nos resultados principalmente para as doses iniciais, sendo mais evidente para o MOSFET canal $n$. Segundo Smedes (1991) [22] e Tsividis e McAndrew (2011) [23], a redução de $g_{m}$ ocasionando o surgimento dos picos observados nas curvas $g_{m} \times V_{G S}$ está associada a dependência não linear da mobilidade dos elétrons em relação ao campo elétrico transversal na região de porta do MOSFET.

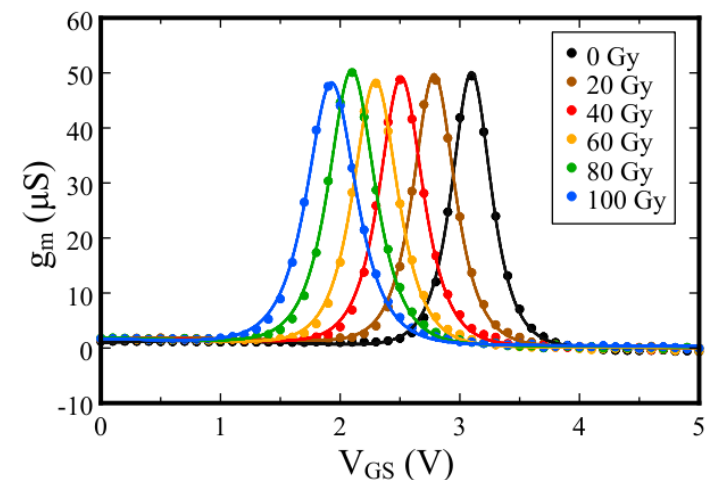

(a)

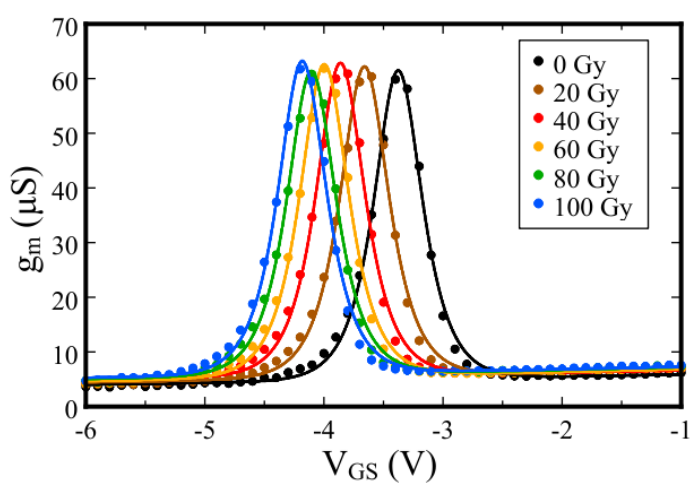

(c)

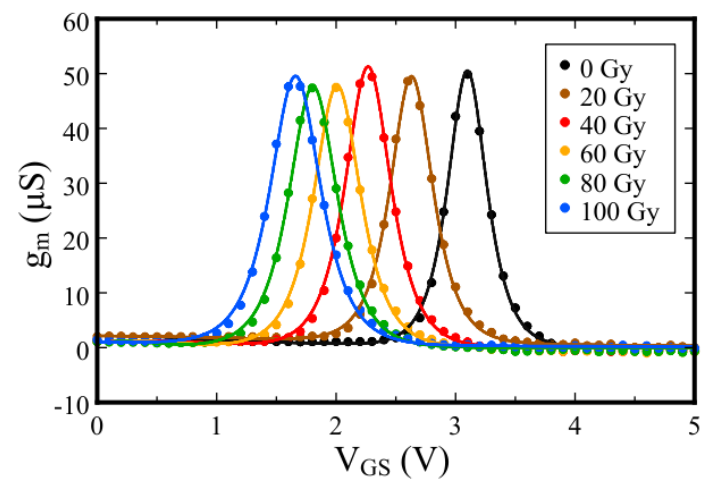

(b)

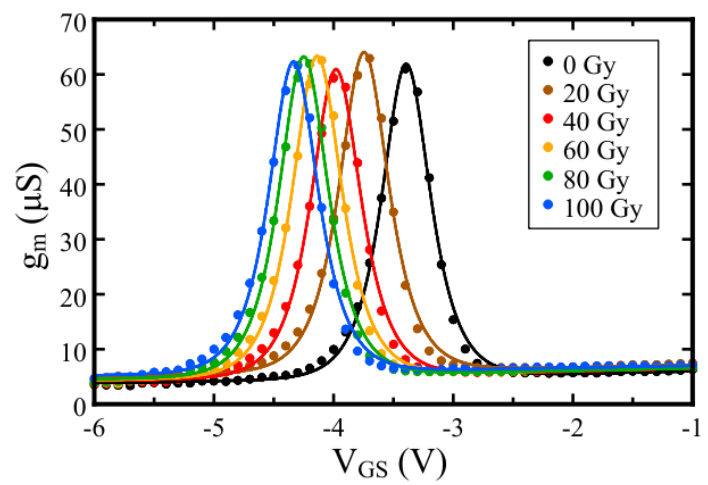

(d)

Figura 4: Curvas $g_{m} \times V_{G S}$ parametrizadas em função da dose de raios X para os MOSFETs: (a) FQP20N06 não polarizado durante as irradiações, (b) FQP20N06 polarizado durante as irradiações, (c) FQP17P10 não polarizado durante as irradiações e (d) FQP17P10 polarizado durante as irradiações. 
A Tabela 2 apresenta os valores da tensão $V_{G S}$ referentes ao valor máximo de $g_{m}$ em função da dose acumulada de radiação, sendo tais resultados extraídos a partir dos parâmetros de uma curva de ajuste não linear obtida por meio do software LabPlot $2^{\circledR}$. Os valores de $V_{G S}$ contidos na Tabela 2 correspondem a média aritmética dos valores encontrados para três amostras irradiadas em cada uma das condições especificadas de polarização elétrica dos MOSFETs. Os desvios padrão nos valores apresentados na Tabela 2 são referentes à média aritmética das medições em triplicata. No intervalo de dose de 0 a 100 Gy tem-se, em valores absolutos, o deslocamento total dos picos de $g_{m}$ de $1,27 \mathrm{~V}$ e $1,46 \mathrm{~V}$ para o MOSFET FQP20N06 e de $0,82 \mathrm{~V}$ e 0,94 V para o MOSFET FQP17P10, respectivamente nas condições não polarizado e sob polarização durante as irradiações. Desses resultados para $g_{m}$ conclui-se que o MOSFET FQP20N06 (canal $n$ ) apresentou maior sensibilidade a radiação ionizante. Nota-se também que a sensibilidade de $V_{G S}$ em função da dose é, aproximadamente, $0,3 \mathrm{~V}$ por cada $20 \mathrm{~Gy}$ de dose, o que implica em cerca de $15 \mathrm{mV} / \mathrm{Gy}$. Isso conduz a concluir que se pode mensurar doses com resolução melhor que $100 \mathrm{mGy}$, uma vez que multímetros modernos podem mensurar pelo menos $1 \mathrm{mV}$ com precisão de $0,1 \mathrm{mV}$.

Tabela 2: Tensão $V_{G S}(V)$ referente ao valor máximo de $g_{m}$ em função da dose de raios $X$.

\begin{tabular}{ccccc}
\hline & \multicolumn{4}{c}{ Condições de polarização elétrica dos MOSFETs durante as irradiações } \\
\cline { 2 - 5 } Dose (Gy) & $\begin{array}{c}\text { FQP20N06 não } \\
\text { polarizado }\end{array}$ & $\begin{array}{c}\text { FQP20N06 } \\
\text { polarizado }\end{array}$ & $\begin{array}{c}\text { FQP17P10 não } \\
\text { polarizado }\end{array}$ & $\begin{array}{c}\text { FQP17P10 } \\
\text { polarizado }\end{array}$ \\
\hline $\mathbf{0}$ & $3,09 \pm 0,01$ & $3,10 \pm 0,01$ & $-3,37 \pm 0,01$ & $-3,39 \pm 0,01$ \\
$\mathbf{2 0}$ & $2,74 \pm 0,04$ & $2,63 \pm 0,01$ & $-3,68 \pm 0,01$ & $-3,71 \pm 0,01$ \\
$\mathbf{4 0}$ & $2,44 \pm 0,06$ & $2,27 \pm 0,01$ & $-3,88 \pm 0,02$ & $-3,98 \pm 0,01$ \\
$\mathbf{6 0}$ & $2,19 \pm 0,09$ & $2,02 \pm 0,01$ & $-4,02 \pm 0,02$ & $-4,14 \pm 0,01$ \\
$\mathbf{8 0}$ & $1,98 \pm 0,11$ & $1,81 \pm 0,01$ & $-4,12 \pm 0,02$ & $-4,25 \pm 0,01$ \\
$\mathbf{1 0 0}$ & $1,82 \pm 0,11$ & $1,64 \pm 0,02$ & $-4,19 \pm 0,02$ & $-4,33 \pm 0,01$ \\
\hline
\end{tabular}

Monte e Santos (2020) [24] apresentaram resultados para $g_{m}$ referentes aos MOSFETs tipo FQD20N06 (canal $n$ ) e FQD17P06 (canal $p$ ) que têm encapsulamento DPAK. Os transistores analisados foram submetidos as mesmas condições de irradiação adotadas neste trabalho. Apesar dos MOSFETs FQP20N06 e FQD20N06 terem características elétricas semelhantes, há duas diferenças básicas em suas características: (1) termodinâmica; (2) mecânica. Ambas as diferenças resultam do tipo de encapsulamento diferente. Primeiramente, por se tratar de um MOSFET que pode ser usado em circuitos de potência (corrente e tensão altas), o modelo FQP possui um dissipador de calor cujo volume é 17 vezes superior ao modelo FQD. Em segundo lugar, as dimensões mecânicas do encapsulamento TO-220 (modelo FQP) quando comparado ao DPAK (modelo FQD) observa-se que a espessura do encapsulamento de cada modelo é, do ponto de vista da interação da radiação ionizante, significativamente diferente, pois apenas alguns $\mu \mathrm{m}$ de espessura resulta no efeito build-up significativamente diferente. Informações detalhadas sobre as dimensões mecânicas desses encapsulamentos podem ser consultadas em [25, 26]. Nos dois tipos de encapsulamentos a pastilha (chip) de silício tem espessura de aproximadamente $300 \mu \mathrm{m}$ e está internamente localizada sobre o substrato metálico. Nos experimentos realizados a face irradiada dos transistores corresponde a face oposta ao substrato metálico. De acordo com as informações contidas em $[25,26]$ e levando em consideração a espessura da pastilha de silício, observa-se que no encapsulamento TO-220 a distância entre a face irradiada e a pastilha é de 2,8 $\mathrm{mm}$, enquanto no encapsulamento DPAK essa distância é de $1,5 \mathrm{~mm}$. O fato é que essa distância, que é quase o dobro no encapsulamento TO-220, é fundamental no efeito build-up do feixe de fótons como mencionado por Gonçalves Filho et al. (2018) [15].

Comparando-se os resultados obtidos para $g_{m}$ apresentados neste trabalho para o MOSFET FQP20N06 com os resultados apresentados por Monte e Santos (2020) [24] para o MOSFET FQD20N06, tem-se que no intervalo de dose de 0 a $100 \mathrm{~Gy}$, o deslocamento total dos picos de $g_{m}$ foi de $1,27 \mathrm{~V}$ e $1,46 \mathrm{~V}$ para o MOSFET FQP20N06 e de 1,41 V e 1,64 V para o MOSFET FQD20N06, respectivamente nas condições não polarizado e polarizado durante as irradiações. 
Estes resultados demonstraram que o efeito build-up no encapsulamento devido às diferentes espessuras dos encapsulamentos proporcionou diferentes resultados em relação ao parâmetro $g_{m}$.

Os resultados obtidos para a capacitância $C_{g d}$ estão apresentados na Figura 5. Observando-se os conjuntos de curvas percebe-se que para o MOSFET FQP20N06, $C_{g d}$ aumenta gradativamente com a dose acumulada de radiação nas duas condições de polarização. Por outro lado, efeito inverso é verificado para o MOSFET FQP17P10. Para os dois modelos de transistores verifica-se maior sensibilidade à radiação ionizante quando os transistores são submetidos à polarização durante as irradiações. Nota-se que maiores variações de $C_{g d}$ ocorreram principalmente para a tensão $V_{D S}$ na faixa de valores de $0 \mathrm{a} \pm 10 \mathrm{~V}$. Observa-se que à medida que a tensão $V_{D S}$ aumenta, a capacitância $C_{g d}$ diminui devido à expansão da região de depleção no semicondutor [27], tornando menos evidente a influência do dano causado pela radiação ionizante sobre $C_{g d}$. As alterações nos valores de $C_{g d}$ devido à incidência da radiação ionizante estão associadas ao aumento de cargas positivas aprisionadas na região do óxido de porta, produzindo alterações no dielétrico do óxido e mudanças nas características elétricas da região de interface $\mathrm{SiO}_{2}-\mathrm{Si}$ [18-20, 28]. A Tabela 3 apresenta resultados para a capacitância $C_{g d}$ na tensão $V_{D S}= \pm 10 \mathrm{~V}$. Os desvios padrão nos valores apresentados na Tabela 3 são referentes à média aritmética das medições em triplicata. Apesar dos resultados até aqui apresentados corresponderem à irradiação com feixes de raios $\mathrm{X}$ aplicados ao radiodiagnóstico, nada impede que o método seja aplicado em feixes de radioterapia, uma vez que a parte ativa do transistor (região $\mathrm{SiO}_{2}-\mathrm{Si}$ do MOSFET) tem apenas algumas dezenas de nanômetros de espessura e por isso o efeito é praticamente semelhante [21].

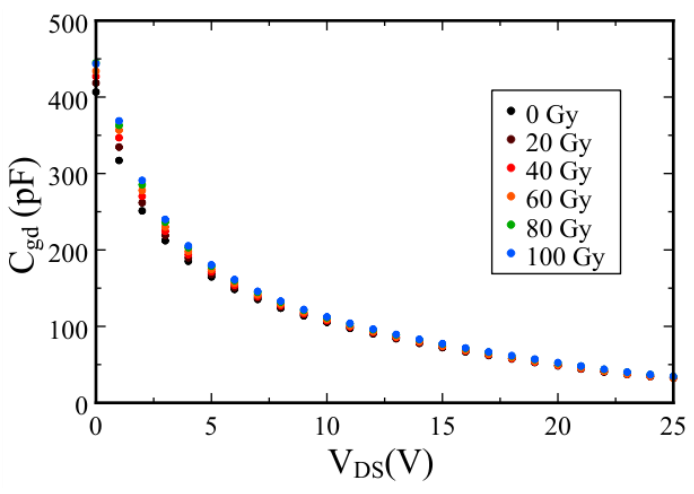

(a)

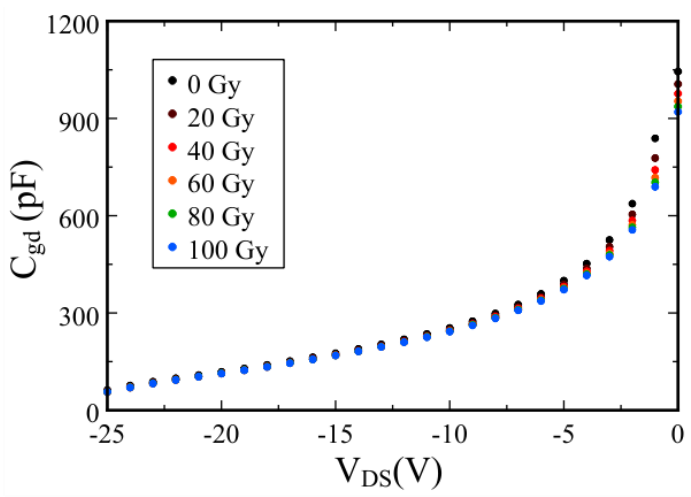

(c)

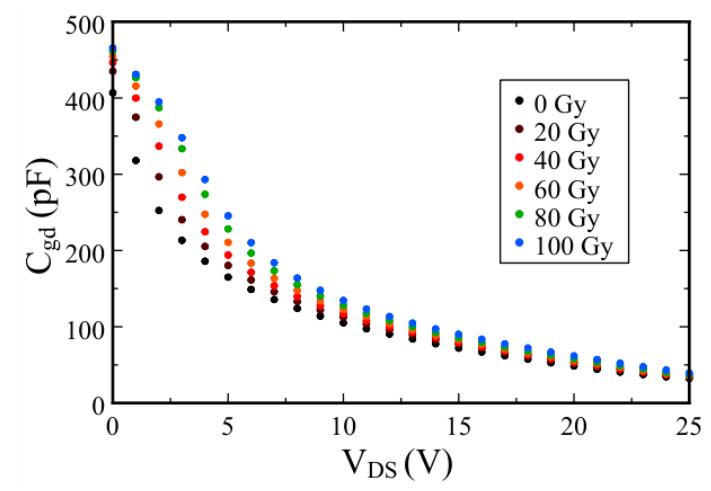

(b)

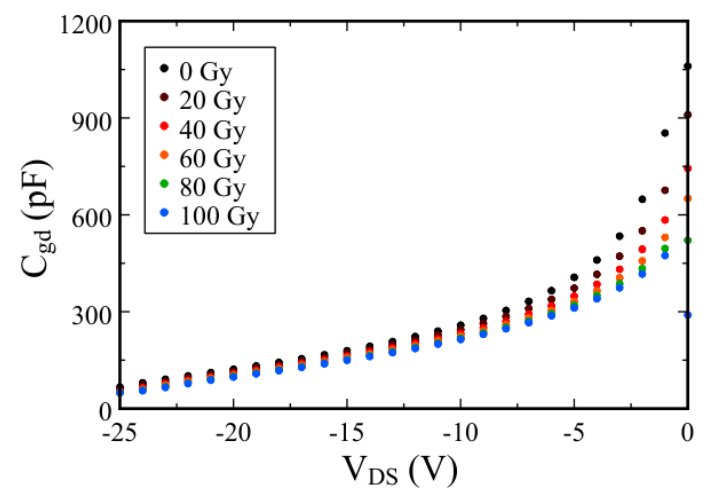

(d)

Figura 5: Curvas $C_{g d} \times V_{D S}$ parametrizadas em função da dose de raios X para os MOSFETs: (a) FQP20N06 não polarizado durante as irradiações, (b) FQP20N06 polarizado durante as irradiações, (c) FQP17P10 não polarizado durante as irradiações e (d) FQP17P10 polarizado durante as irradiações. 
Tabela 3:Capacitância $C_{g d}(p F)$ para a tensão $V_{D S}= \pm 10$ V em função da dose de raios $X$.

\begin{tabular}{ccccc}
\hline & \multicolumn{3}{c}{ Condições de polarização elétrica dos MOSFETs durante as irradiações } \\
\cline { 2 - 5 } $\begin{array}{c}\text { Dose } \\
(\mathbf{G y})\end{array}$ & $\begin{array}{c}\text { FQP20N06 não } \\
\text { polarizado }\end{array}$ & $\begin{array}{c}\text { FQP20N06 } \\
\text { polarizado }\end{array}$ & $\begin{array}{c}\text { FQP17P10 não } \\
\text { polarizado }\end{array}$ & $\begin{array}{c}\text { FQP17P10 } \\
\text { polarizado }\end{array}$ \\
\hline $\mathbf{0}$ & $105,19 \pm 0,77$ & $105,08 \pm 0,57$ & $249,06 \pm 0,81$ & $257,26 \pm 0,67$ \\
$\mathbf{2 0}$ & $106,49 \pm 0,97$ & $112,52 \pm 0,95$ & $244,61 \pm 0,79$ & $243,69 \pm 0,45$ \\
$\mathbf{4 0}$ & $107,67 \pm 0,30$ & $117,06 \pm 0,68$ & $242,05 \pm 0,89$ & $231,68 \pm 0,44$ \\
$\mathbf{6 0}$ & $108,91 \pm 0,29$ & $122,56 \pm 0,73$ & $240,04 \pm 0,68$ & $223,23 \pm 0,56$ \\
$\mathbf{8 0}$ & $111,54 \pm 0,77$ & $128,17 \pm 0,35$ & $240,19 \pm 0,48$ & $217,05 \pm 0,28$ \\
$\mathbf{1 0 0}$ & $112,52 \pm 0,39$ & $134,39 \pm 0,76$ & $238,94 \pm 0,45$ & $213,61 \pm 0,74$ \\
\hline
\end{tabular}

Monte e Santos (2020) [24] também apresentaram resultados para a capacitância $C_{g d}$ referentes aos MOSFETs série FQD20N06 (canal $n$ ) e série FQD17P06 (canal $p$ ) que possuem encapsulamento DPAK. Comparando-se aos resultados deste trabalho, constatou-se que a capacitância $C_{g d}$ demonstrou comportamento semelhante para os dois tipos de encapsulamentos e polaridades dos MOSFETs. No caso dos MOSFETs canal $p$, em termos de características elétricas, eles diferem em relação à tensão $V_{D S}$ máxima suportada $(100 \mathrm{~V}$ para o FQP17P10 e $60 \mathrm{~V}$ para o FQD17P06), entretanto a tendência de comportamento da capacitância $C_{g d}$ em relação à dose de radiação acumulada ainda é mantida para os dois tipos de transistores.

Em se tratando da capacitância $C_{d s}$, os resultados obtidos estão apresentados na Figura 6 e demonstraram que este parâmetro não apresentou sensibilidade à radiação ionizante para a dose acumulada na faixa de 0 a 100 Gy. De acordo com Leroy e Rancoita (2007) [21], a predominância de danos causados ao MOSFET pela radiação ionizante está associada as regiões de óxido (danos de ionização). Visto que a capacitância $C_{d s}$ não está associada as regiões de óxido, estando localizada entre as regiões do dreno e da fonte internamente a estrutura do MOSFET [27, 29], a baixa sensibilidade à radiação ionizante apresentada por esse parâmetro mostra-se como resultado coerente.

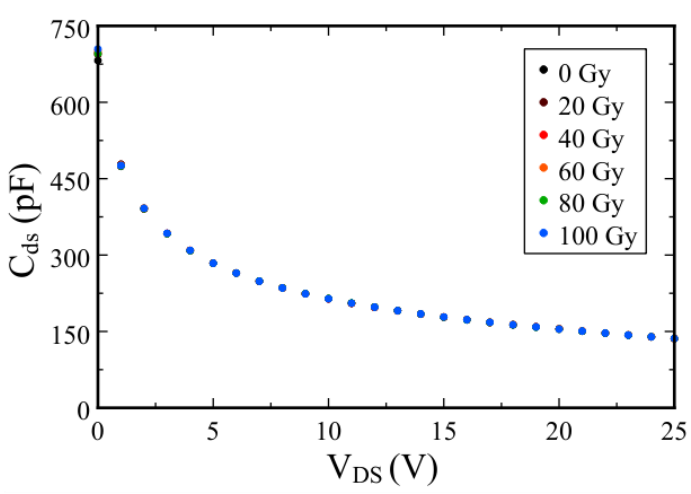

(a)

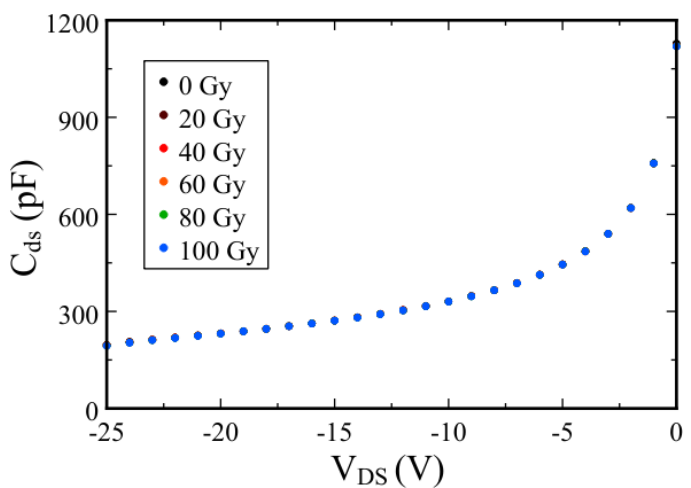

(c)

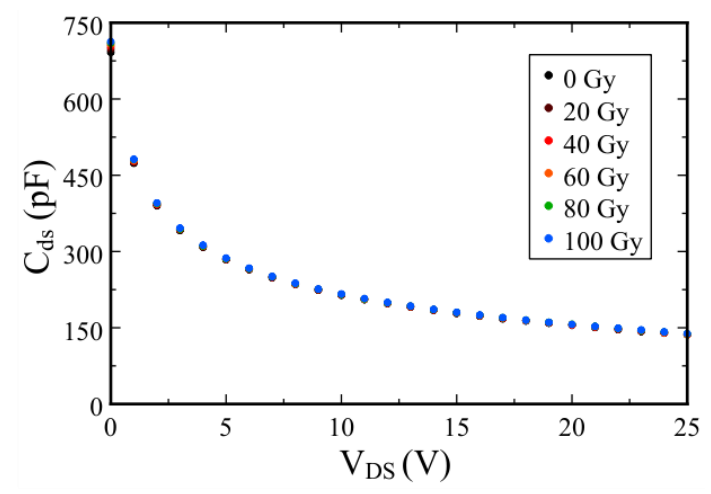

(b)

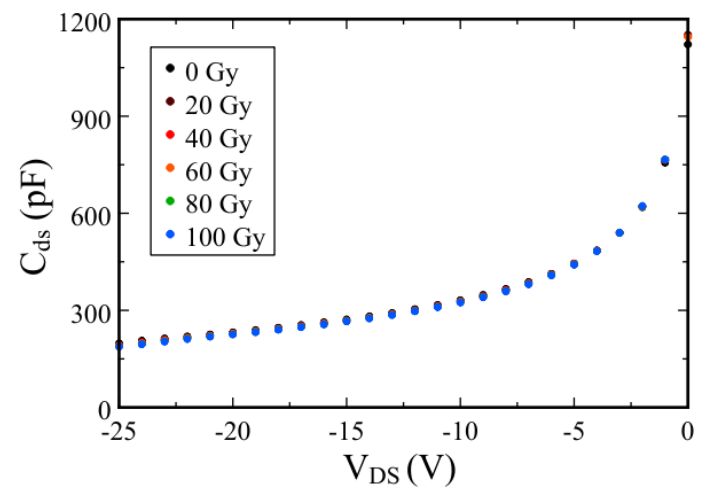

(d)

Figura 6: Curvas $C_{d s} \times V_{D S}$ parametrizadas em função da dose de raios X para os MOSFETs: (a) FQP20NO6 não polarizado durante as irradiações, (b) FQP20N06 polarizado durante as irradiações, (c) FQP17P10 não polarizado durante as irradiações e (d) FQP17P10 polarizado durante as irradiações. 


\section{CONCLUSÃO}

Este trabalho teve como objetivo apresentar o efeito de um feixe de raios X, na faixa de energia que é aplicado ao diagnóstico médico, em um MOSFET visando analisar a variação de alguns parâmetros $\pi$-híbridos para então correlacionar com a dose de radiação recebida pelo dispositivo.

Do ponto de vista do problema inverso, essa técnica inovadora indicou que a medição da dose de radiação pode ser feita por meio da variação dos parâmetros $\pi$-híbridos do MOSFET. Com base nos resultados obtidos, o método apresentado apontou que os parâmetros avaliados $g_{m}$ e $C_{g d}$ têm sensibilidade à radiação ionizante e podem ser utilizados para correlacionar com a dose de radiação ionizante recebida pelo MOSFET. Em contrapartida, o parâmetro $C_{d s}$ não apresentou variação perceptível mediante incidência da radiação ionizante. Em relação à polarização elétrica do transistor durante as irradiações, ela mostrou-se como um fator diferencial inclusive melhorando a sensibilidade dos parâmetros $g_{m}$ e $C_{g d}$.

Do ponto de vista de inovação ao estado da técnica no processo de mensuração da dose de raios $\mathrm{X}$ aplicados ao diagnóstico médico usando um MOSFET, por meio de um parâmetro diferente do convencional, o objetivo foi atingido. Embora a faixa de energia do feixe de radiação adotado enquadre-se na faixa do que é aplicado ao radiodiagnóstico, nada impede que o método seja aplicado em feixes de radioterapia. Assim, este trabalho poderá servir como referência para o desenvolvimento científico e tecnológico na área de dosimetria de radiação ionizante.

\section{AGRADECIMENTOS}

Agradecemos a Luiz Carlos Gonçalves Filho pelo suporte técnico durante as irradiações, e ao CNPq (Proc. 315215/2018-6) e a CAPES (Proc. 88882.379353/2019-1) pelo apoio financeiro aos pesquisadores.

\section{REFERÊNCIAS BIBLIOGRÁFICAS}

1. Gray PR, Hurst PJ, Lewis SH, Meyer RG. Analysis and Design of Analog Integrated Circuits. 5. ed. New York: John Wiley \& Sons; 2009.

2. Sedra AS, Smith KC. Microelectronic Circuits. 7. ed. New York: Oxford University Press; 2015.

3. Hughes RC, Huffman D, Snelling JV, Zipperian TE, Ricco AJ, Kelsey CA. Miniature radiation dosimeter for in vivo radiation measurements. Int J Radiat Oncol Biol Phys. 1988 Mai;14(5):963-967, doi: 10.1016/0360-3016(88)90019-3.

4. Sarrabayrousse G, Siskos S. Radiation dose measurements using MOSFETs. IEEE Instrum Meas Mag. 1998 Jun;1(2):26-34, doi:10.1109/5289.685494.

5. Rosenfeld AB. Electronic dosimetry in radiation therapy. Radiat Meas. 2006 Dez;4(Suppl 1):S134-S153, doi: 10.1016/j.radmeas.2007.01.005.

6. Banqueri J, Carvajal MA, Martinez-Garcia MS, Morales DP, Palma AJ. Study of the subthreshold swing of a pMOSFET as a dosimetric parameter. Sens Actuator A-Phys. 2012 Nov;187:16-21, doi: 10.1016/j.sna.2012.08.015.

7. Martinez-Garcia MS, Simancas F, Palma AJ, Lallena AM, Banqueri J, Carvajal MA. General purpose MOSFETs for the dosimetry of electron beams used in intra-operative radiotherapy. Sens Actuator APhys. 2014 Abr;210(1):175-181, doi: 10.1016/j.sna.2014.02.019.

8. Kumar AS, Sharma SD, Ravindran BP. Characteristics of mobile MOSFET dosimetry system for megavoltage photon beams. J Med Phys. 2014 Jul;39(3):142-149, doi: 10.4103/0971-6203.139002.

9. Sah C-T. Fundamentals of solid-state electronics. Singapore: World Scientific; 2006.

10. Rashid MH. Power electronic handbook: Devices, circuits, and applications. 3. ed. Burlington: Elsevier; 2011.

11. Bertling K, Rakic AD, Yeow YT, Brawley A, Domyo H, Rotella FM. Comparison of SOS MOSFET's equivalent circuit parameters extracted from LCR meter and VNA measurement. IEEE Trans Electron Devices. 2012 Jan;59(1):20-25, doi:10.1109/TED.2011.2170426.

12. Direct Power MOSFET Capacitance Measurement at $3000 \mathrm{~V}$ - Application Note. Keysight Technologies Inc., U.S.A., Dez. 2017. [Online]. Disponível em: http://literature.cdn.keysight.com/litweb/pdf/59907145EN.pdf 
13. Kakitani H, Takeda R. Precise Evaluation of Input, Output, and Reverse Transfer Capacitances of Power Devices - White Paper. Keysight Technologies International Japan G.K., USA, Out. 2014. [Online]. Disponível em: http://literature.cdn.keysight.com/litweb/pdf/5992-0207EN.pdf

14. Su J-G, Wong S-C, Chang C-Y, Huang T-Y. The extraction of MOSFET gate capacitance from Sparameter measurements. Solid-state Electronics. 2002 Ago;46(8):1163-1167, doi: 10.1016/S00381101(02)00022-9.

15. Goncalves Filho LC, Monte DS, Barros FR, Santos LAP. Radiation dose response of n-channel MOSFET submitted to filtered $x$-ray photon beam. IEEE Trans Nuclear Sci. 2018 Set;65(9):2607-2610, doi:10.1109/TNS.2018.2846668.

16. Santos LAP, Cavalcanti FA, Nobrega GS, Monte DS, Araújo GG, Alves AN, Silva JO, Santos MAP. Techniques for measuring some characteristics of ionizing radiation beams using bipolar junction transistor as a detector. In: 3rd International Conference on Advancements in Nuclear Instrumentation, Measurement Methods and their Applications (ANIMMA); 23-27 Jun 2013; Marseille, France. [local desconhecido]: IEEE; 2014. [5 p.], doi:10.1109/ANIMMA.2013.6728091.

17. Srour JR, Marshall CJ, Marshall PW. Review of displacement damage effects in silicon devices. IEEE Trans Nuclear Sci. 2003 Jun;50(3):653-670, doi: 10.1109/TNS.2003.813197.

18. Fleetwood DM. Evolution of total ionizing dose effects in MOS devices with moore's law scaling. IEEE Trans Nuclear Sci. 2018 Ago;65(8):1465-1481, doi:10.1109/TNS.2017.2786140.

19. Schwank JR, Shaneyfelt MR, Fleetwood DM, Felix JA, Dodd PE, Paillet P, Ferlet-Cavrois, V. Radiation effects in MOS oxides. IEEE Trans Nuclear Sci. 2008 Ago;55(4):1833-1853, doi: 10.1109/TNS.2008.2001040.

20. Lutz G. Semiconductor Radiation Detectors: Device Physics. 1. ed. New York: Springer; 1999.

21. Leroy C, Rancoita PG. Particle interaction and displacement damage in silicon devices operated in radiation environments. Rep Progr Phys. 2007 Mar;70(4):493-625, doi: 10.1088/0034-4885/70/4/R01.

22. Smedes T. Compact modelling of the dynamic behaviour of MOSFETs. Eindhoven: Technische Universiteit Eindhoven; 1991.

23. Tsividis Y, Mcandrew C. Operation and modeling of the MOS transistor. 3. ed. New York: Oxford University Press; 2011.

24. Monte DS, Santos LAP. A method for measuring ionizing radiation dose by analyzing hybrid- $\pi$ parameters of transistors. In: IEEE International Instrumentation and Measurement Technology Conference (I2MTC); 25-28 Maio 2020; Dubrovnik, Croatia. [local desconhecido]: IEEE; 2020. [6 p.], doi:10.1109/I2MTC43012.2020.9129059.

25. Mechanical Case Outline - Package Dimensions - TO-220-3LD. Semiconductor Components Industries, LLC, 2019. [Online]. Disponível em: https://www.onsemi.com/pub/Collateral/340AT.PDF.

26. Mechanical Case Outline - Package Dimensions - DPAK3 (TO-252 3 LD). Semiconductor Components Industries, LLC, 2019. [Online]. Disponível em: https://www.onsemi.com/pub/Collateral/369AS.PDF.

27. Baliga BJ. Fundamentals of power semiconductor devices. 2. ed. New Delhi: Springer; 2019.

28. Spieler H. Semiconductor detector system. New York: Oxford University Press; 2005. (Series on Semiconductor Science and Technology).

29. Tietze U, Schenk C, Gamm E. Electronic circuits: Handbook for design and application. 2. ed. Heidelberg: Springer; 2008. 